\title{
Efectos cardiovasculares del tabaquismo
}

\author{
Carolina Reyes-Méndez, Cinthia Fierros-Rodríguez, Ramiro Cárdenas-Ledesma, \\ Andrea Hernández-Pérez, $\bowtie$ Leonor García-Gómez, Rogelio Pérez-Padilla
}

Instituto Nacional de Enfermedades Respiratorias Ismael Cosío Villegas, Ciudad de México, México.

\begin{abstract}
RESUMEN. El consumo de tabaco tiene consecuencias adversas en la salud cardiovascular y representa un problema de salud pública a nivel mundial que demanda atención integral. Se realizó una revisión sistemática en PubMed de la literatura concerniente al impacto del tabaquismo sobre la salud cardiovascular reportada en metaanálisis publicados en los últimos 10 años. Doce estudios determinaron una asociación significativa entre el tabaquismo y la incidencia de enfermedades cardiovasculares, de los cuales seis demostraron una disminución importante de la morbimortalidad tras la implementación de políticas para el control del tabaco. Es necesario fortalecer las acciones de los gobiernos y los profesionales de la salud para disminuir la prevalencia de consumo de tabaco y proteger a la población general de las enfermedades asociadas con este producto, que afectan a todo el cuerpo y que tienden a generar enfermos con múltiples comorbilidades que se tienen que investigar.
\end{abstract}

Palabras clave: Tabaquismo, enfermedad cardiovascular, legislación para control de tabaco.

\section{Cardiovascular effects of smoking}

ABSTRACT. Cigarette smoking has hazardous effects on cardiovascular health and represents a worldwide great concern that demands further attention. We conducted a systematic review using PubMed database of the literature concerning cardiovascular effects of cigarette smoking reported in meta-analyses published in the last 10 years. 12 studies determined a significant association between cigarette smoking and the incidence of cardiovascular diseases, 6 of them demonstrated a substantial decline in the morbimortality after tobacco control policies were implemented. There is a need for the governments and the health care providers to strengthen their actions in order to reduce the prevalence of tobacco smoking and protect the general population from smoking attributable diseases, affecting multiple organs and systems and generating patients with multiple comorbidities.

Key words: Cigarette smoking, cardiovascular disease, tobacco control policies.

\section{INTRODUCCIÓN}

El consumo de tabaco es el principal factor de riesgo individual prevenible a nivel global, causando más de 7 millones de muertes prematuras anuales, de las cuales 900,000 se derivan de la exposición al humo de segunda mano. Asimismo, es factor de riesgo de 6 de las 8 principales causas de muerte a nivel mundial, y ocasiona 1 de cada 6 decesos por enfermedades no transmisibles. En México se estima que la prevalencia de consumo de tabaco es de $16.4 \%$ en población de 15

\section{$\triangle$ Autor para correspondencia:}

Dra. Andrea Hernández Pérez, Instituto Nacional de Enfermedades Respiratorias Ismael Cosío Villegas, Ciudad de México, México.

Correo electrónico: andrea.hde@gmail.com

Trabajo recibido: 21-IX-2018; aceptado: 27-XI-2018 años y más, equivalente a 14.3 millones de fumadores, mientras la cifra anual de fallecimientos por enfermedades atribuibles al tabaquismo se calcula en 43,000 personas, representando el $8.4 \%$ del total de muertes en el país. ${ }^{1}$ El Instituto Nacional de Salud Pública reporta un gasto de 75 mil millones de pesos al año en atención de enfermedades relacionadas con el tabaquismo. ${ }^{2}$

El principal componente psicoactivo del tabaco es la nicotina, una sustancia que actúa en el sistema nervioso central generando cambios bioquímicos responsables de la adicción. Aunado a ello, más de 7,000 sustancias 
químicas están presentes en el humo, cientos de las cuales son tóxicas y potencialmente capaces de generar daño sistémico. ${ }^{3}$ Debido a que al menos 69 componentes son carcinógenos conocidos, la exposición al mismo aumenta el riesgo de padecer diversos tipos de cáncer, enfermedades pulmonares y otros problemas de salud graves entre los que figuran las enfermedades cardiovasculares (ECV). ${ }^{4}$

Las ECV son la principal causa de muerte en todo el mundo y el consumo de tabaco es el segundo factor de riesgo predominante, sólo después de la hipertensión arterial sistémica. Particularmente, es un factor de riesgo importante de cardiopatía coronaria, ECV y vasculopatía periférica. Tanto el consumo de tabaco, como la exposición al humo ajeno contribuyen a cerca del $17 \%$ de las defunciones por cardiopatías. ${ }^{4}$ Cada año la Organización Mundial de la Salud (OMS) y sus asociados celebran el Día Mundial sin Tabaco con el fin de destacar los riesgos asociados con el tabaquismo y abogar por políticas eficaces para reducir su consumo. Si bien los daños a la salud cardíaca provocados por el tabaco y los tratamientos para reducir la morbimortalidad que ocasiona han sido identificados, amplios sectores de la población desconocen que el tabaquismo es una de las principales causas de las ECV. Es por ello que en 2018 la OMS enfatizó las repercusiones del consumo de tabaco en la salud cardiovascular de las personas en todo el mundo. Además, la campaña busca atender posibles medidas y acciones que los gobiernos y el público en general pueden adoptar para reducir los riesgos a la salud derivados del consumo de tabaco. Por lo que en el presente artículo se presenta una revisión de la evidencia disponible de los efectos cardiovasculares del tabaquismo, así como los resultados obtenidos con la implementación de la legislación para el control del tabaco.

\section{MÉTODO}

Se llevó a cabo una búsqueda sistemática en PubMed con los criterios de búsqueda [tobacco smoking] and [cardiovascular diseases] de mayo a agosto de 2018, restringiendo los resultados únicamente a metaanálisis publicados en los últimos 10 años (2008-2018). Se encontraron 35 artículos, de los cuales 12 fueron seleccionados por especificar la relación entre el tabaquismo y efectos cardiovasculares.

\section{Resultados de la búsqueda}

\section{Efectos cardiovasculares del tabaquismo}

Existe evidencia contundente de que fumar incrementa el riesgo de desarrollar ECV, ${ }^{5}$ y que tanto la exposición de manera activa o pasiva al humo de tabaco son causas de morbilidad y mortalidad cardiovascular. El monóxido de carbono, los químicos oxidantes y la nicotina juegan un papel fundamental en el desarrollo y progresión de diversas afecciones, presumiblemente debido al daño endotelial e incremento en la agregación plaquetaria. La nicotina en particular ejerce un efecto excitatorio en el sistema nervioso simpático que conlleva un aumento en la presión arterial y frecuencia cardíaca a corto plazo, con mayor trabajo y estrés cardíaco; sin embargo, dicho efecto no explica toda la asociación a largo plazo entre el tabaquismo y las ECV. ${ }^{5}$

El tabaquismo se relaciona con diversos mecanismos que precipitan la aparición de trombosis, hemorragia o vasoconstricción, mismos que derivan en oclusión vascular e isquemia, entre los que se incluyen un menor suministro de sangre oxigenada al miocardio y el consecuente incremento en el gasto cardíaco. ${ }^{3,6}$ Otro aspecto clave en la patogénesis de ECV inducidas por tabaquismo es la disfunción endotelial en arterias coronarias y periféricas, ya que se produce un estado inflamatorio crónico que contribuye a los procesos de enfermedad aterogénica y eleva los niveles de biomarcadores de inflamación, conocidos predictores de eventos cardiovasculares. Asimismo, se generan anormalidades en el perfil lipídico que predisponen al desarrollo de aterosclerosis, principalmente debido a un aumento en los triglicéridos y una disminución en el colesterol asociado a lipoproteínas de alta densidad. El consumo de cigarro también condiciona resistencia a la insulina e inflamación crónica, lo que puede acelerar las complicaciones micro y macrovasculares, incluida la nefropatía. ${ }^{7}$ En este sentido, numerosos estudios sugieren una relación entre el tabaquismo y la incidencia de diabetes, obesidad y síndrome metabólico. ${ }^{5}$

Existe un mayor riesgo de IAM entre los usuarios de productos de tabaco en comparación con quienes no fuman. ${ }^{5}$ Muchos fumadores piensan que disminuir la cantidad de cigarros que consumen reduce sustancialmente el riesgo de desarrollar enfermedades asociadas al consumo; si bien disminuir la cantidad de cigarros fumados al día puede aminorar el riesgo de padecer cáncer y algunas afecciones cardiovasculares, está comprobado que fumar relativamente poco (1 a 5 cigarros por día) se relaciona con un riesgo de ECV y ACV más alto de lo que muchos profesionales de la salud o fumadores reconocen. ${ }^{6}$

Un estudio identificó que los hombres que fuman un cigarro al día tienen un incremento del $48 \%$ en el riesgo de padecer enfermedades cardíacas, en comparación con los que nunca han fumado, mientras que para las mujeres fumadoras el riesgo es $57 \%$ más grande en comparación con mujeres sin historial de tabaquismo. 
Particularmente, en los hombres, el riesgo relativo de presentar ECV es de 1.48 para los consumidores de un cigarro al día y de 2.04 para los fumadores de 20 cigarros al día; en las mujeres, el riesgo relativo es de 1.57 para quienes fuman un cigarro al día, mientras que para las que fuman 20 el riesgo asciende a 2.84. Los riesgos relativos incrementan en los consumidores de grandes cantidades de cigarro al ajustar múltiples factores, entre los que se encuentran un mayor índice de masa corporal, adiposidad y dietas insalubres. En general, fumar un sólo 1 cigarro por día conlleva un riesgo importante de desarrollar ECV que se calcula entre el 40 y $50 \%$ del riesgo, asociado con el consumo de 20 cigarros diarios. ${ }^{6}$

Una revisión sistemática de 75 cohortes que incluyeron datos de 2.4 millones de individuos y más de 44,000 eventos coronarios en total sugiere que, en comparación con no fumadores, las mujeres fumadoras presentan un riesgo relativo $25 \%$ mayor de ECV que los hombres fumadores, independientemente de otros factores de riesgo cardiovascular ( $R R 1.25,95 \% \mathrm{Cl} 1.12-$ $1.39, p<0$ 0001); además, se calculó que las mujeres presentaron un incremento en el riesgo de $2 \%$ por cada año de seguimiento $(p=0.03)$. También se examinó la asociación entre el tabaquismo y ECV en fumadores actuales e individuos sin antecedentes ni consumo actual de tabaquismo agrupados por rangos de edad (de los 30 a los 80 años); en todas las categorías, con excepción de los más jóvenes (30 a 44 años), el efecto del tabaquismo en el riesgo de EC fue más grande en mujeres que en hombres, aunque la diferencia sólo fue significativa para los individuos de 60 a 69 años de edad. El análisis de los datos obtenidos en 53 estudios no encontró diferencias entre hombres y mujeres ex fumadores en comparación con los que nunca habían fumado respecto al riesgo de ECV (RR $0.96,95 \% \mathrm{Cl}$ $0.86-1.08, p=0.53$ ); no está claro si la probabilidad de ECV para cada sexo se atenúa o se incrementa conforme aumenta la edad. Si bien la mayoría de los estudios ha reportado un mayor riesgo en mujeres fumadoras que en hombres fumadores para desarrollar ECV, no está claro si los mecanismos que subyacen dicha diferencia son biológicos o están relacionados con la conducta tabáquica entre hombres y mujeres. La exposición a partículas y a otros tóxicos, por ejemplo, puede ser mayor en mujeres que en hombres al fumar un cigarrillo al día, ya que en general el tamaño corporal es menor. Se enfatiza que los programas de control de tabaco deben dirigirse con la misma intensidad a las mujeres, sobre todo en aquellos países donde está incrementando la prevalencia de tabaquismo en mujeres jóvenes. ${ }^{8}$

Entre los fumadores, el riesgo de SICA se reduce unos días después de dejar de fumar. ${ }^{9}$ Se estima una reducción del $36 \%$ en el riesgo de mortalidad por cualquier causa en los pacientes con ECV que dejan de fumar en comparación con los que continúan fumando, misma que parece ser constante independientemente de la edad, el sexo y el índice de eventos cardíacos. ${ }^{10}$

La American Cancer Society señala que 1 de cada 10 defunciones por eventos cardiovasculares son atribuibles al tabaquismo, específicamente debidas a IAM y $\mathrm{ACV},{ }^{11}$ y que el tabaquismo pasivo también es un factor de riesgo importante para el IAM; se ha calculado que la exposición al humo de segunda mano incrementa el riesgo en un 25 a $31 \% .{ }^{9}$ La eliminación del humo de segunda mano reduce su incidencia, lo cual cobra sentido en términos biológicos, ya que la exposición puede inducir rápidamente cambios fisiopatológicos importantes de acuerdo con un estudio que comparó a fumadores activos y no fumadores en los procesos de agregación plaquetaria, trombosis, disfunción endotelial e inflamación. Se estimó que dichos efectos aumentan el riesgo de IAM en alrededor del $10 \%$ a nivel mundial. ${ }^{12}$ Siguiendo esta línea de investigación, otro estudio corroboró que en los no fumadores incluso una breve exposición se asocia con efectos adversos en la agregación plaquetaria, elasticidad vascular, función endotelial, variabilidad del ritmo cardíaco, y metabolismo de lípidos, lo cual sustenta la necesidad biológica de restricciones de consumo de tabaco., ${ }^{9,20}$

Dentro de las afecciones más severas del tabaquismo en el sistema nervioso central se destaca el ACV, que al igual que otras ECV, es una de las principales causas de discapacidad y muerte en el mundo.,13

Numerosos estudios evidencian la relación causal entre el tabaquismo y las enfermedades cerebrovasculares. Tras ajustar otros factores de riesgo, los fumadores tienen un mayor riesgo de presentar ACV y mayores índices de mortalidad derivada del mismo en comparación con individuos que nunca han fumado. ${ }^{3}$

En un amplio estudio se identificó que los hombres que fuman 1 cigarro al día tienen un riesgo incrementado en $25 \%$ de presentar ACV en comparación con los que nunca han sido fumadores, mientras que en las mujeres fumadoras el incremento en el riesgo se estima en $31 \%$ en comparación con mujeres sin historial de tabaquismo. Específicamente, se encontró que el riesgo relativo de ACV en hombres es de 1.25 para fumadores de un cigarro diario y de 1.64 para fumadores de 20 cigarros al día; en las mujeres se calculó en 1.31 y 2.16 para las fumadoras de un cigarro y de 20 cigarros, respectivamente. Aun teniendo en cuenta múltiples factores de confusión se reconoce que los fumadores empedernidos tienden a presentar más factores severos de riesgo cardiovascular que los fumadores «leves» (de cantidades bajas de cigarro). ${ }^{6}$ 
El consumo de cigarro de asocia con un mayor riesgo para todos los subtipos de eventos cerebrovasculares. $1^{3}$ Se ha puntualizado una fuerte relación dosis-respuesta tanto para el ACV isquémico como para la hemorragia subaracnoidea. ${ }^{7,14}$

Un estudio internacional en una muestra de 26,919 participantes reclutados de 32 países que comparó la importancia de los factores de riesgo para cada subtipo de ACV a partir de su incidencia señala que el tabaquismo se asocia más significativamente con ACV de tipo isquémico que con hemorragia intracerebral ( $p$ $<0.0001){ }^{13}$

Al respecto, otro estudio indica que en participantes para quienes la presencia de tabaquismo era el único factor de riesgo, la incidencia de ACV isquémico fue mayor en hombres que en mujeres, con una menor sintomatología en comparación con participantes que presentaban otros factores de riesgo. Además, se observó que el primer evento ocurrió en edades más tempranas en los participantes fumadores en comparación con participantes no fumadores. ${ }^{15}$

Otra evidencia importante es el Estudio del Corazón de Framingham, que evaluó el impacto del tabaquismo en la incidencia de ACV en una cohorte 4,255 hombres y mujeres de 36 a 68 años de edad. Durante 26 años de seguimiento se reportaron 459 accidentes cerebrovasculares; la incidencia anual fue $23.3 \%$ más grande en hombres que en mujeres. El accidente isquémico transitorio representó el $15 \%$ del total de eventos, mientras que el infarto isquémico aterotrombótico representó el $52.9 \%$ y su incidencia fue $32.3 \%$ mayor en hombres que en mujeres. Independientemente del grado de tabaquismo y el sexo, los participantes con hipertensión presentaron el doble de incidencia de ACV. Empleado el método de regresión de Cox y considerando otros factores de riesgo cardiovascular como la edad y la hipertensión, el tabaquismo resultó ser un factor que contribuye de manera independiente y significativa al riesgo de presentar $A C V$ en general, específicamente de tipo isquémico. Se observó una correlación positiva entre el riesgo relativo de ACV y el número de cigarros fumados; particularmente, el riesgo en fumadores de más de 40 cigarros al día fue el doble del riesgo en fumadores de menos de 10 cigarros al día. Se calculó que el riesgo se redujo significativamente tras dos años de abstinencia y que se equiparó al de los no fumadores dentro de los primeros cinco años después de la cesación, ${ }^{16}$ lo cual corresponde con varios estudios, observaciones que han demostrado que el incremento en el riesgo de sufrir ACV por tabaquismo disminuye al de dejar de fumar y desaparece tras cinco años en abstinencia. ${ }^{17,18}$

Un estudio prospectivo exploró los factores de riesgo de ACV en una cohorte de 10,938 individuos normo- tensos $(<140 / 90 \mathrm{mmHg}$ ); se reportaron 56 eventos cerebrovasculares, de los cuales se estimó que el $39 \%$ fueron atribuibles al tabaquismo. Se realizó un análisis multivariado que incluyó otros factores de riesgo típicos (edad, obesidad, antecedentes de ECV), de los cuales el tabaquismo tuvo un alto riesgo relativo (RR, 3.21; $95 \% \mathrm{Cl}, 1.82$ a 5.66); además de la edad, fue el factor de riesgo responsable de la mayoría de los eventos. ${ }^{19}$ Asimismo, los estudios indican una asociación fuerte, consistente y dependiente de la dosis entre la exposición a humo de segunda mano y el riesgo de presentar ACV, suficiente para inferir una relación causal ${ }^{20}$ caracterizada por un incremento en el riesgo del 20 al $30 \%$. $^{3}$

Dado que no existe un nivel seguro de consumo en el que pueda asumirse que fumar no causará daños a la salud cerebrovascular, los fumadores «leves» necesitan dejar de fumar por completo si desean disminuir significativamente el riesgo de presentar ACV. ${ }^{6}$

\section{Reducción de las EC con la legislación para el control del tabaco}

Se prevé que el consumo de tabaco sea responsable de la defunción de cerca de mil millones de personas en todo el mundo durante el siglo XXI. ${ }^{12} \mathrm{~A}$ pesar de que en años recientes se han alcanzado muchos logros en la protección de la salud de la población frente al tabaco, la carga de enfermedad causada por su consumo, así como los altos costos humanos y económicos han motivado que organismos internacionales implementen diversos planes de acción para reducir la mortalidad prematura y la prevalencia de consumo actual mediante la prevención, tratamiento y promoción de la salud y el bienestar.

En este sentido, la evidencia consistente de los efectos perjudiciales del tabaquismo ha motivado a muchos países a promulgar diversas legislaciones con el fin de disminuir directamente la exposición al humo de tabaco ambiental y reducir indirectamente el tabaquismo activo con el fin de prevenir y reducir los costos sanitarios y económicos de la enfermedad. ${ }^{1}$ Se ha comprobado que la legislación de espacios libres de humo de tabaco a nivel local, estatal y nacional, tanto en espacios públicos cerrados como en lugares de trabajo, incluyendo restaurantes y bares, se asocia con un menor riesgo de hospitalización y muerte derivadas de eventos coronarios (ECV, SICA e IAM), otras cardiopatías (angina y muerte súbita) y ACV (infarto cerebral incluyendo el subtipo transitorio). ${ }^{3}$

En particular, la implementación de leyes exhaustivamente restrictivas se asocia con índices significativamente más bajos de admisiones hospitalarias o muertes en población con diagnóstico de eventos coronarios (RR 
0.848, IC 95\%: 0.816-0.881), ACV (RR 0.840, IC 95\%: $0.753-0.936$ ), enfermedades respiratorias (RR 0.760 , IC 95\%: 0.682-0.846) y otras cardiopatías (RR 0.610, IC 95\%: 0.440-0.847). ${ }^{21}$

Los estudios indican que la incidencia de IAM en la población general disminuyó de manera significativa tras la legislación de espacios libres de humo de tabaco, usualmente dentro de un período de tiempo corto (RR 0.87, IC 95\%: 0.84-0.91).12,22 Se ha reportado específicamente que la incidencia de IAM disminuyó en un 33\% $(p<0.001)$ y la incidencia de muerte súbita disminuyó en un $17 \%(p=0.13)$ en los 18 meses siguientes a la instauración de políticas de lugares de trabajo libres de humo en comparación con los 18 meses anteriores a la implementación de legislación antitabáquica. ${ }^{23}$ Otro estudio corrobora que las prohibiciones de fumar en espacios públicos y laborales se asocian significativamente con una reducción en la incidencia de IAM (RR 0.83, IC 95\%: 0.75-0.92), particularmente si se aplica durante varios años, y que dicha asociación se ve reforzada por otros factores entre los que se encuentran altos niveles de cumplimiento de tales políticas, disminución de la prevalencia y venta de tabaco, mejor calidad de aire y una menor exposición ambiental al humo de tabaco. ${ }^{9}$ El efecto benéfico de la prohibición de fumar parece ser rápido, con disminuciones en la incidencia de IAM dentro de los tres primeros meses, y es más pronunciado en los individuos jóvenes y los no fumadores. ${ }^{9}$

De hecho, se ha identificado que las medidas más restrictivas de consumo de tabaco en espacios públicos cerrados se relacionan con la disminución del riesgo de desarrollar distintas enfermedades asociadas al tabaquismo. Algunos investigadores lo consideran como una respuesta dependiente de la dosis en la que, entre más restrictivas sean las medidas respecto al consumo de tabaco, menor es el riesgo relativo de presentar eventos coronarios y otras afecciones cardíacas, ACV y enfermedades respiratorias. Es importante notar que las leyes por sí mismas no producen los efectos observados, sino que éstos derivan tanto de la reducción de la exposición a humo de segunda mano como del incremento en la cesación tabáquica que acompañan a estas leyes. La adopción de políticas de ambientes libres de humo refleja cambios en las normas sociales que también afectan la conducta de fumar, y las leyes tanto formalizan como aceleran este cambio de perspectiva social y sus consecuentes beneficios a la salud. ${ }^{21}$

El efecto más significativo en la reducción en las tasas de admisión a urgencias por enfermedades relacionadas con el tabaquismo durante el período de 1999 a 2005 en Ohio, Estados Unidos, se observó en la ECV, donde la incidencia se redujo significativamente en un $39 \%$ después de 1 año y en un $47 \%$ después de 3 años posteriores a la implementación de legislaciones de espacios libres de humo de tabaco. ${ }^{24}$

La evidencia muestra de manera contundente que la instauración de leyes que pugnan por frenar la exposición a humo de tabaco ambiental causa una mejora en la salud de la población y, a su vez, una reducción en los costos que implica para los sistemas sanitarios enfrentar la carga de enfermedad asociada al tabaquismo. Es importante mantener una supervisión continua del cumplimiento de las legislaciones vigentes, así como alentar a los países que aún no adoptan políticas públicas tendientes a restringir el consumo activo y pasivo de tabaco a implementarlas con carácter urgente. ${ }^{22}$

\section{CONCLUSIONES}

El tabaquismo es un factor de riesgo modificable. Numerosos estudios han comprobado que la implementación de políticas de control de tabaco reduce la morbimortalidad cardiovascular, estimula el cese del consumo en los fumadores, concientiza a la población general sobre los efectos adversos del tabaquismo y reduce la exposición ambiental al humo de tabaco ajeno entre los fumadores pasivos. Se trata de una ardua labor que exige esfuerzos conjuntos, por lo que los resultados podrían ser más pronunciados a largo plazo. ${ }^{12}$

Existe evidencia contundente para afirmar que no basta con disminuir el consumo activo de tabaco para reducir el riesgo de ECV, sino que se requiere erradicarlo por completo al igual que al humo de tabaco de segunda mano, pues tampoco existen límites seguros de exposición, ${ }^{20}$ por lo que la necesidad de proteger a los no fumadores es imperante.

Las metas globales que los organismos internacionales han trazado para controlar la epidemia de tabaquismo y las devastadoras consecuencias sociales y económicas que causa exigen el fortalecimiento de las legislaciones restrictivas de consumo, así como el establecimiento de acuerdos, estrategias de monitoreo, vigilancia y evaluación de las normas antitabaco. Sin duda la expansión de las políticas de control de tabaco tiene una importancia crucial para la salud pública, y tanto los responsables de su implementación a nivel legislativo como los profesionales de la salud deben pugnar por medidas más severas e integrales que prohíban fumar en áreas públicas y lugares de trabajo. ${ }^{12}$

Todos los integrantes del equipo clínico en cualquier nivel de atención deben alentar su cumplimiento y realizar un trabajo multidisciplinario para abordar uno de los principales factores de riesgo de ECV: el tabaquismo. Desalentar su consumo es un objetivo central y un de- 
safío importante que implica acciones terapéuticas de calidad; particularmente en la población usuaria de los servicios de sanitarios, una valoración médica integral que implique la intervención del cardiólogo desde los ámbitos de prevención, detección, diagnóstico y tratamiento puede ser una aportación significativa para hacer frente a estas problemáticas de salud.

Es importante señalar que los nuevos productos de tabaco como el cigarro electrónico no han comprobado su eficacia ni seguridad; contienen nicotina, componente activo que no sólo es causante de su uso, abuso y adicción, sino también de alteraciones vasculares que pueden, en última instancia, comprometer la salud cardiovascular.

También que los enfermos respiratorios con consecuencias adversas del tabaquismo, deben ser evaluados para la presencia de otras enfermedades, como las cardiovasculares o cerebrovasculares, también causadas por el tabaquismo, situación que predice la presencia cada vez más marcada de enfermos con múltiples comorbilidades, que van a dificultar el entrenamiento del personal de salud y el control de las mismas. La mejor solución es luchar meticulosamente contra el tabaquismo en todas sus formas.

\section{Conflicto de intereses}

Los autores declaran no tener conflicto de intereses.

\section{REFERENCIAS}

1. Reynales-Shigematsu LM, Zavala-Arciniega L, PazBallesteros WC, et al. Encuesta Nacional de Consumo de Drogas, Alcohol y Tabaco 2016-2017: Reporte de Tabaco. Ciudad de México, México: INPRFM; 2017.p.19,20.

2. Instituto Nacional de Salud Pública (2013). 75 mil millones de pesos al año gasta México por tabaquismo. Fecha de consulta: 20 de agosto 2018. Accesible en: https://www.insp.mx/centros/salud-poblacional/ prensa/1345-75-mil-millones-de-pesos-al-ano-gastamexico-por-tabaquismo.html

3. U.S. Department of Health and Human Services. The health consequences of smoking - 50 years of progress: a report of the Surgeon General. Atlanta, GA: U.S. Department of Health and Human Services, Centers for Disease Control and Prevention, National Center for Chronic Disease Prevention and Health Promotion, Office on Smoking and Health; 2014.p.413-435.

4. Organización Mundial de la Salud. Campañas mundiales de salud pública de la OMS. Día mundial sin tabaco 2018: Tabaco y cardiopatías. Fecha de consulta: 30 de mayo, 2018. Accesible en: http://www.who.int/ campaigns/no-tobacco-day/2018/event/es/

5. Boffetta P, Straif K. Use of smokeless tobacco and risk of myocardial infarction and stroke: systematic review with meta-analysis. BMJ 2009;339:b3060. doi: 10.1136/ bmj.b3060.

6. Hackshaw A, Morris JK, Boniface S, Tang JL, Milenković D. Low cigarette consumption and risk of coronary heart disease and stroke: meta-analysis of 141 cohort studies in 55 study reports. BMJ 2018;360:j5855. doi: 10.1136/ bmj.j5855.

7. U.S. Department of Health and Human Services. How tobacco smoke causes disease: the biology and behavioral basis for smoking attributable-disease: a report of the surgeon general. Atlanta, GA: U.S. Department of Health and Human Services, Centers for Disease Control and Prevention, National Center for Chronic Disease Prevention and Health Promotion, Office on Smoking and Health; 2010.p.11,361.

8. Huxley RR, Woodward M. Cigarette smoking as a risk factor for coronary heart disease in women compared with men: a systematic review and meta-analysis of prospective cohort studies. Lancet 2011;378(9799):12971305. doi: 10.1016/S0140-6736(11)60781-2.

9. Meyers DG, Neuberger JS, He J. Cardiovascular effect of bans on smoking in public places: a systematic review and meta-analysis. J Am Coll Cardiol 2009;54(14):12491255. doi: 10.1016/j.jacc.2009.07.022.

10. Critchley JA, Capewell S. Mortality risk reduction associated with smoking cessation in patients with coronary heart disease: a systematic review. JAMA 2003;290(1):86-97. doi: 10.1001/jama.290.1.86.

11. Ezzati M, Henley SJ, Thun M J, Lopez AD. Role of smoking in global and regional cardiovascular mortality. Circulation 2005;112(4):489-497. doi: 10.1161/ CIRCULATIONAHA.104.521708.

12. Lin $\mathrm{H}$, Wang $\mathrm{H}, \mathrm{Wu}$ W, Lang $\mathrm{L}$, Wang Q, Tian L. The effects of smoke-free legislation on acute myocardial infarction: a systematic review and meta-analysis. MBC Public Health 2013;13:529. doi: 10.1186/1471-2458-13529.

13. O'Donnell MJ, Chin SL, Rangarajan S, et al. Global and regional effects of potentially modifiable risk factors associated with acute stroke in 32 countries (INTERSTROKE): a case-control study. Lancet 2016;338(10046):761-775. doi: 10.1016/ S01406736(16)30506-2.

14. Ockene IS, Miller NH. Cigarette smoking, cardiovascular disease, and stroke: a statement for healthcare professionals from the American Heart Association. American Heart Association Task Force on Risk Reduction. Circulation 1997;96(9):3243-3247.

15. Fukuoka T, Nakazato $Y$, Kawasaki H, et al. The clinical features of ischemic stroke patients for whom smoking was considered the sole risk factor for ischemic stroke. Intern Med 2018;57(12):1703-1706. doi: 10.2169/ internalmedicine.9655-17.

16. Wolf PA, D'Agostino RB, Kannel WB, Bonita R, Belanger AJ. Cigarette smoking as a risk factor for stroke. The Framingham Study. JAMA 1988;259(7):1025-1029. doi: 10.1001/jama.1988.03720070025028.

17. Kawachi I, Colditz GA, Stampfer MJ, et al. Smoking cessation and decreased risk of stroke 
in women. JAMA 1993;269(2):232-236. 10.1001/ jama.1993.03500020066033.

18. Wannamethee SG, Shaper AG, Whincup PH, Walker $\mathrm{M}$. Smoking cessation and the risk of stroke in middleaged men. JAMA 1995;274(2):155-160. doi: 10.1001/ jama.1995.03530020073035.

19. Li C, Engström G, Hedblad B, Berglund G, Janzon L. Risk factors for stroke in subjects with normal blood pressure: a prospective cohort study. Stroke 2005;36(2):234-238. doi: 10.1161/01.STR.0000152328.66493.0a.

20. Oono IP, Mackay DF, Pell JP. Meta-analysis of the association between secondhand smoke exposure and stroke. J Public Health (Oxf) 2011;33(4):496-502. doi: 10.1093/pubmed/fdr025.

21. Tan CE, Glantz SA. Association between smokefree legislation and hospitalizations for cardiac, cerebrovascular, and respiratory diseases: a metaanalysis. Circulation 2012;126(18):2177-2183. doi: 10.1161/CIRCULATIONAHA.112.121301.

22. Mackay DF, Irfan MO, Haw S, Pell JP. Meta-analysis of the effect of comprehensive smoke-free legislation on acute coronary events. Heart 2010;96(19):1525-1530. doi: 10.1136/hrt.2010.199026.

23. Hurt RD, Weston SA, Ebbert JO, et al. Myocardial infarction and sudden cardiac death in Olmsted County, Minnesota, before and after smoke-free workplace laws. Arch Intern Med 2012;172(21):1635-1641. doi: 10.1001/2013.jamainternmed.46.

24. Khuder SA, Milz S, Jordan T, Price J, Silvestri K, Butler $\mathrm{P}$. The impact of a smoking ban on hospital admissions for coronary heart disease. Prev Med 2007;45(1):3-8. doi: 10.1016/j.ypmed.2007.03.011. 